\title{
Exposição ocupacional do cirurgião-dentista à vibração mecânica transmitida através das mãos: um estudo de caso
}

\author{
Gilsée Ivan Regis Filho, ${ }^{\mathrm{a}, *}$, Thiago Rodrigo Lóss Zmijevskib, Louise Pietrobonc, \\ Marianella Aguilar Ventura Fadel ${ }^{\mathrm{d}}$, Francisco Keller Klug ${ }^{\mathrm{e}}$ \\ a,**gregis@brturbo.com.br, UFSC, Brasil \\ btzmijevski@yahoo.com, UFSC, Brasil \\ cisepietro@terra.com.br, UFSC, Brasil \\ ${ }^{d}$ maya@ccs.ufsc.br, UFSC, Brasil \\ efranciscokk@gmail.com, UFSC, Brasil
}

\begin{abstract}
Resumo
O objetivo do presente trabalho foi estimar a exposição a vibrações induzidas pelo uso de instrumentos rotatórios por cirurgiões-dentistas. Os instrumentos rotatórios analisados foram de alta e de baixa rotação. Os efeitos deletérios das vibrações no conjunto mão/braço são uma função da frequência e da amplitude do sinal. Observou-se que os efeitos dependem, ainda, da excitação ser constituída por uma única frequência ou apresentar um espectro complexo. Foram testadas três amostras de cada instrumento rotatório (canetas), a saber: caneta de alta rotação, contra-ângulo de baixa rotação e peça de mão, também de baixa rotação. A utilização por cirurgiões-dentistas dos instrumentos rotatórios avaliados aqui não representa risco ocupacional em termos das vibrações transmitidas às mãos, mesmo que operados continuamente ao longo de seis horas de trabalho; entretanto pode ser importante fator coadjuvante no desenvolvimento de LERs/DORTs. Por outro lado, o conjunto de efeitos fisiológicos das vibrações leva a crer que fortes oscilações mecânicas diminuem a capacidade de desempenho e em muitas situações aumentam o risco de falhas e acidentes.
\end{abstract}

Palavras-chave

Vibrações. Exposição ocupacional. Cirurgião-dentista.

\section{Introdução}

A exposição diária e repetida a vibrações no local de trabalho pode causar modificações patológicas das partes do corpo envolvidas. 0 tipo de patologia é diferente para as duas partes do corpo mais sujeitas às vibrações: a coluna vertebral e os membros superiores. As oscilações verticais que penetram no corpo de quem está sentado ou de pé sobre bases vibratórias, como veículos, levam geralmente a manifestações de desgaste na coluna, enquanto as oscilações de ferramentas motorizadas geram na maioria das vezes manifestações patológicas nas mãos e braços (GRANDJEAN, 1998).

Tarefasefatoresno trabalho têmsidoidentificados como responsáveis pelo desenvolvimento da síndrome do túnel carpal. São eles: movimentos repetitivos de alta velocidade do punho e dedos, com carga sobre os tendões no túnel do carpo, contração forcada destes tendões, extrema flexão ou extensão do punho, trabalho muscular estático, posições extremas da mão no trabalho, posição desajeitada, estresse mecânico do nervo mediano na base da palma, luvas mal ajustadas e, ainda, as vibrações (LUOPAJARVl et al., 1979).

Constata-se que, nos Estados Unidos, além de cerca de 52\% dos relatos de dores nas costas serem atribuídos pelo trabalhador a eventos repetitivos no trabalho (GU0 et al., 1995), 24\% das desordens de longa duração no dorso estavam relacionados ao movimento de se curvar e erguer, ao trabalho em posturas inadequadas e à operação de máquinas que vibram. Além do que $8 \%$ da população estavam expostos a pelo menos dois desses três fatores e outros 3\% a todos os três (LIIRA et al. 1996).

Os efeitos deletérios das vibrações no conjunto mão/braço são uma função da frequência e da amplitude do sinal. Observou-se que os efeitos 
dependem, ainda, da excitação ser constituída por uma única frequência ou apresentar um espectro complexo. Todos os estudos mostraram que não há variações sensíveis em função das variáveis extrínsecas, tais como a massa do indivíduo, o tamanho, a postura e a tensão muscular, excetuando-se a tensão com a qual a alavanca é fixada (NEPOMUCENO, 1984).

Viikari-Juntura et al. (1994) selecionaram grupos de trabalhadores para um estudo longitudinal baseado em diferentes exposições ao trabalho. Operadores de máquinas expostos ao trabalho estático e a vibrações no corpo inteiro foram comparados com carpinteiros que realizam um trabalho físico dinâmico e presumivelmente sob nenhuma vibração, para observar se a condição ocupacional estava relacionada com lesões por Esforços Repetitivos (LERs) do pescoço. Os resultados mostraram que a taxa de probabilidade para progredir de nenhuma dor no pescoço até problemas moderados a severos nessa região ia de 3,9 a 4,2 vezes mais para os operadores de máquinas que transmitiam vibrações - diferença bem significativa.

Entretanto, para Bernard (1997), nenhum estudo determinou claramente uma associação entre força, vibração e Lesões por Esforços Repetitivos/Distúrbios Osteomusculares Relacionados ao Trabalho (LERs/ DORTs) do pescoço, não havendo, assim, evidência suficiente para apoiar qualquer ligação entre vibração e LERs/DORTs de pescoço ou pescoço/ ombro com base em critérios de força.

Burdorf e Monster (1991) e Stenlund et al. (1992, 1993) desenvolveram estudos com diversas categorias profissionais, como perfuradores de rochas e colocadores de rebites, objetivando verificar uma associação entre LERs/DORTs ou dor no ombro e vibração produzida por ferramentas ou máquinas, no entanto não há evidência suficiente para uma ligação entre lesão de ombro e exposição à vibração da região.

0 mecanismo pelo qual vibração contribui para a síndrome do túnel carpal não é bem compreendido, provavelmente, porque a exposição à vibração é geralmente acompanhada por exposição a movimentos forçados e repetitivos (BERNARD, 1997). Para o autor, músculos expostos à vibração exibem um reflexo tônico de vibração que causa uma intensa contração muscular involuntária. Por outro lado, está demonstrado que a vibração produz prejuízos à capacidade táctil, em curto prazo, podendo levar a um aumento da quantidade de força exercida durante tarefas manuais. A vibração também pode levar à abrasão mecânica das bainhas dos tendões e lesionar diretamente os nervos periféricos e os terminais nervosos, produzindo sintomas de amortecimento, formigamento, dor e perda de sensibilidade.

Lundborg et al. (1987) constataram que em ratos a vibração causa edema epineural no nervo ciático. Assim, parece haver evidência razoável suportando uma associação entre exposição à vibração e síndrome do túnel carpal.

A vibração da mão/braço geralmente é definida como a transferência de vibração de uma ferramenta para a mão e braço de um trabalhador. A quantidade de vibração é caracterizada pelo nível de aceleração da ferramenta quando empunhada pelo operário e durante o uso. A vibração é tipicamente medida no cabo da ferramenta, enquanto em uso, para determinar os níveis de aceleração transferidos para o trabalhador. A vibração transmitida para as mãos é produzida por diversos processos na indústria, agricultura, mineração, construção e em serviços (BERNARD, 1997).

A exposição ocupacional à vibração transmitida para as mãos e dedos pode induzir o desenvolvimento de diversos distúrbios, tais como circulatórios, dos ossos e articulações, musculares e outros, bem como do sistema nervoso central (GRIFFIN, 1982, citado por CARNICELLI, 1994). Os mecanismos pelo qual a vibração mão/braço produz dano neurológico, vascular e músculo-esquelético são relatados nos estudos de Armstrong et al. (1987), Lundborg et al. (1990) e Necking et al. (1992).

Distúrbios neurológicos e circulatórios ocorrem provavelmente independentemente. A vibração pode lesionar diretamente os nervos periféricos, os terminais nervosos e mecanoreceptores, produzindo sintomas de amortecimento, formigamento, dor e perda de sensibilidade. A vibração pode, ainda, ter efeitos diretos sobre as artérias digitais. A camada mais interna de células nas paredes dos vasos sanguíneos parece, especialmente, suscetível à lesão mecânica por vibração. Se esses vasos são danificados, podem se tornar menos sensíveis às ações de certos vasodilatadores que requerem um endotélio intacto (BERNARD, 1997). Experiências envolvendo madeireiros expostos à vibração de motosserras dão apoio a esta hipótese (GEMNE; LUNDSTROM; HANSSON, 1993).

Há também evidências de que as paredes dos vasos sanguíneos digitais ficam espessadas em pessoas com síndrome da vibração mão/braço (TAKEUCHI et al., 1986). Durante exposição ao frio, os vasos com este espessamento se tornam anormalmente mais estreitos, podendo se fechar inteiramente (GEMNE, 1982).

Outra evidência, com respeito à coerência de informação sobre a associação entre exposição à vibração e síndrome da vibração mão/braço, se 
relaciona à ocorrência de ligação com outras desordens similares na população em geral como, por exemplo, a doença de Raynaud de origem ocupacional, que é um distúrbio vascular periférico no qual o frio desencadeia uma deficiência de suprimento sanguíneo nos dedos ou nas mãos. Esta tem como causa primária a exposição intensa e constante à transferência de vibração de ferramentas para as mãos, porém 0 principal sintoma é a coloração esbranquiçada que os dedos assumem intermitentemente (CARNICELLl, 1994). Uma estimativa colocou a prevalência do fenômeno de Raynaud em 4,6\% para mulheres e 2,5\% para homens na população em geral (IWATA; MAKIMO, 1987).

$\mathrm{Na}$ maioria dos estudos encontra-se taxa de prevalência maior do que 20\% entre trabalhadores expostos à síndrome da vibração mão/braço. Este fato fornece forte evidência de que indivíduos trabalhando em ocupações expostos à vibração se acham sob muito maior risco destas desordens do que aqueles na população em geral para outras patologias similares. Alguns elementos potenciais primários para a síndrome da vibração mão/braço são a idade e doenças do metabolismo (BERNARD, 1997). Para o autor, diversos estudos levam à conclusão de que há substancial evidência de que, conforme aumentam a duração e a intensidade de uso de ferramentas vibratórias, aumenta também o risco de o trabalhador desenvolver síndrome da vibração mão/braço.

Por outro lado, entre as categorias profissionais expostas a um risco considerável de desenvolver LERs/DORTs e que utilizam instrumentos que provocam vibrações encontram-se os cirurgiõesdentistas.

Para Sebastião, Marziale e Robazzi (2007), em estudo que utilizou revisão sistemática da literatura, a população de trabalhadores industriais é a mais acometida por sequelas osteomusculares nervosas e vasculares em função da exposição à vibração. Entretanto, investigações minuciosas deveriam ser realizadas para avaliar o nível de acometimento de outros profissionais menos pesquisados como os cirurgiões-dentistas, já que estes profissionais são expostos a níveis de vibração de alta frequência não consideradas lesivas.

Dasgupta e Harrison (1996 apud SEBASTIÃO; MARZIZLE; ROBAZZI; 2007), relatam que em publicação a respeito das diretivas sobre a exposição à vibração por cirurgiões-dentistas é mostrado que a emissão danosa da frequência de vibração por equipamentos dentários é muito baixa, sendo que dessa forma não houve interesse em estender os critérios das diretivas para o trabalho odontológico.
De acordo com Szymmanska (2001) e Balbinot e Tamagna (2001) (apud SEBASTIÃO; MARZIALE; ROBAZZl, 2007), uma polêmica ainda envolve a questão da exposição quanto ao nível de aceleração, tempo de exposição ao longo do dia e da função, intervalos previstos para descanso, sendo que estudos mostram que equipamentos de baixa frequência, entre 5 e $20 \mathrm{~Hz}$, são potencialmente mais perigosos do que os que emitem alta frequência. Assim, verifica-se também que não se exclui a possibilidade daqueles que lidam com equipamentos dentários desenvolverem sequelas ao longo de anos de exposição, como os cirurgiõesdentistas, de acordo com Nakládová et al. (2005 apud SEBASTIÃO; MARZIALE; ROBAZZI, 2007).

Alguns autores, como Cândido, Bittencourt e Regis Filho (2003), Martins, Fadel e Regis Filho (2003) e Fadel et al. (2006), apresentam relatos de caso de LERs/DORTs, respectivamente, de cisto sinovial de punho, síndrome do túnel carpal e dedo em gatilho em cirurgião-dentista, sendo todos do sexo feminino, em que a prática odontológica contribuiu, decididamente, para o desencadeamento da LER/DORT e que trouxeram forte limitação funcional nos dois últimos casos.

Estudo epidemiológico transversal realizado por Regis Filho, Michels e Sell (2006) indicou associação estatisticamente significante entre os dois sexos para presença de LERs/DORTs, sendo que o sexo feminino apresenta mais patologias que o masculino. Ombro/braço (39,40\%), punho/mão $(18,30 \%)$ e pescoço $(17,20 \%)$ são as regiões mais afetadas por LERs/DORTs.

Por outro lado, em estudo realizado por Regis Filho, Michels e Sell (2005) com cirurgiõesdentistas e utilizando análise biomecânica, os dados cinemáticos revelaram que as principais atividades realizadas pelos profissionais submetem duas ou mais regiões do corpo a posturas consideradas de risco médio e alto de ocorrência de LERs/DORTs, bem como a análise dos sinais eletromiográficos indicou um grande comprometimento dos grupamentos musculares flexores e extensores do carpo e do trapézio, o que explica a maior incidência das LERs/ DORTs nas regiões de ombro/braço e punho/mão.

Para os autores anteriormente citados a análise biomecânica permite comprovar o que pesquisas utilizando estudos epidemiológicos transversais e Análise Ergonômica do Trabalhojá indicavam: que os cirurgiões-dentistas formam um grupo profissional exposto a risco considerável de adquirir algum tipo de LER/DORT, desde que certos fatores inerentes às tarefas profissionais aí consideradas, como força excessiva, postura incorreta, alta repetitividade de um mesmo padrão de movimento e compressão mecânica dos tecidos, aliadas às características individuais, estejam presentes. 
A vibração produzida pelos instrumentos rotatórios utilizados pelos cirurgiões-dentistas pode ser fator coadjuvante importante para o aparecimento de LERs/DORTs nessa categoria profissional, e há uma base significativa de evidências científicas de que devem ser desenvolvidos mais estudos com relação aos efeitos deletérios da vibração transmitidos às mãos pelos instrumentos rotatórios utilizados pelos profissionais da odontologia.

0 objetivo do presente trabalho foi estimar a exposição às vibrações induzidas pelo uso de instrumentos rotatório por cirurgiões-dentistas. Os instrumentos rotatórios analisados foram de alta e de baixa rotação.

\section{Material e métodos}

\subsection{Instrumentos avaliados}

Foram testadas por um cirurgião-dentista três amostras de cada instrumento rotatório denominados canetas, a saber: caneta de alta rotação, contra-ângulo de baixa rotação e peça de mão, também de baixa rotação (Figura1).

\subsection{Medição de vibração localizada}

A cadeia de medição de vibrações foi composta pelos equipamentos da Figura 2.
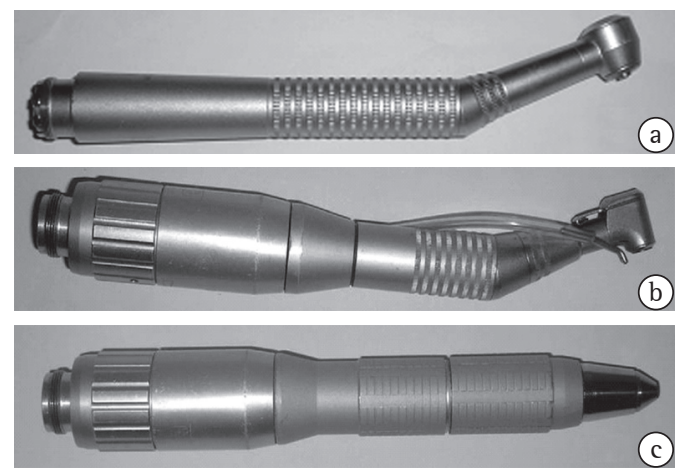

Figura 1. a) Kavo 625N Super Torque (caneta 1), Kavo 605C Extra Torque (canetas 2 e 3), b) Kavo L-motor $181 \mathrm{db}$ (canetas 4, 5 e 6) e c) Kavo peça de mão (canetas 7, 8 e 9).

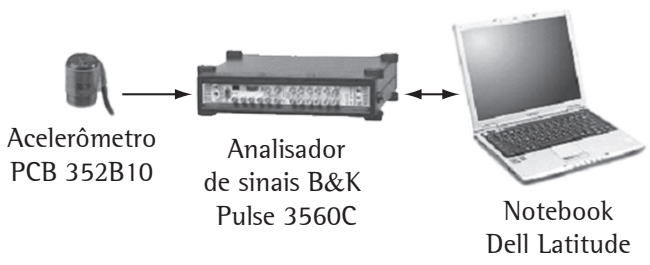

Figura 2. Cadeia de medição.
As medições de vibração seguiram as determinações da norma ISO 5349-1: 2001, partes 1 e 2 (ISO, 2001), sendo realizadas em apenas um eixo. Nesse caso, recomenda-se que a vibração total seja obtida multiplicando-se o valor do eixo de maior magnitude por uma constante que varia entre 1 e 1,7. Foi utilizado um fator de 1,7 para as canetas de alta e baixa rotação, tendo em vista a dificuldade de isolar o eixo de maior vibração, e um fator de 1,4 para a peça de mão, na qual a vibração se sobressai em duas direções de forma equivalente ( $\mathrm{x}$ e z).

0 transdutor (acelerômetro) foi fixado às canetas por meio de cera, nas proximidades dos dedos indicador e polegar do operador. A direção de medição foi paralela ao eixo de rotação da fresa para as canetas de alta e de baixa rotação (Figura 3) e perpendicular para o caso da peça de mão (Figura 4).

0 primeiro passo no processamento dos dados foi expressar a aceleração para o eixo medido em bandas de 1/3 de oitava. A seguir, a aceleração RMS total ponderada em frequência para o eixo é dada pela Equação 1:

$a_{h w(\text { eixo })}=\sqrt{\sum_{i}\left(W_{h i} a_{i}\right)^{2}}$

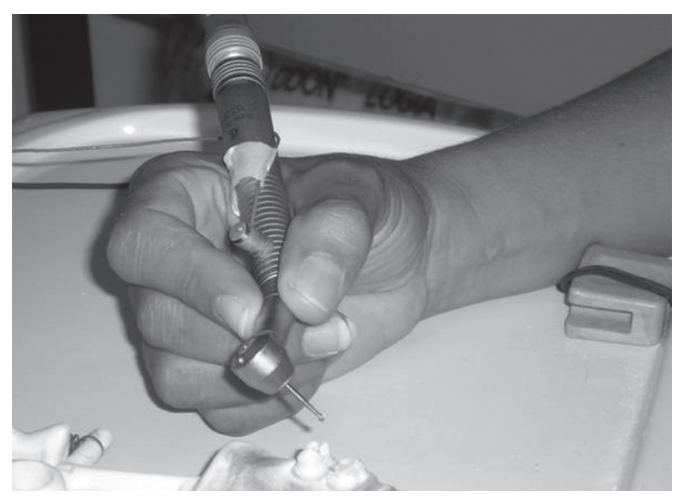

Figura 3. Caneta de alta rotação.

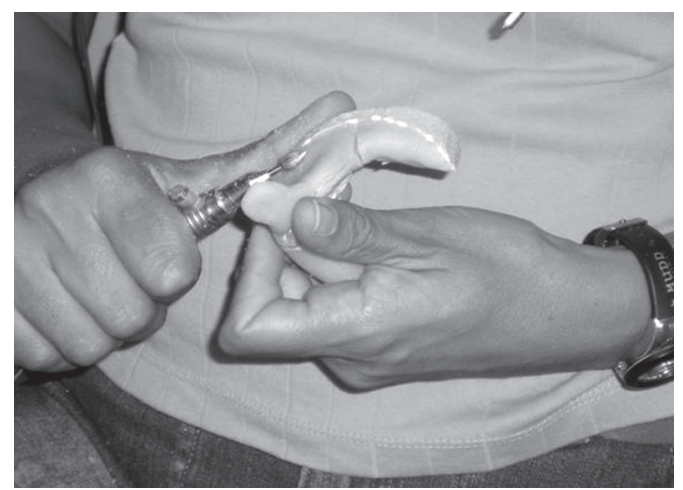

Figura 4. Contra-ângulo de baixa rotação. 
Sendo $W_{h i}$ o fator de ponderação da i-ésima banda de 1/3 de oitava, segundo ISO 5349, e $a_{i}$ a aceleração RMS medida na respectiva banda.

0 nível global de vibrações é obtido multiplicando-se por um fator $\kappa$ a aceleração RMS total ponderada em frequência para o eixo (Equação 2). Como comentado, adotou-se um k de 1,7 para as canetas de alta rotação e baixa rotação e 1,4 para o contra-ângulo:

$a_{h v}=k \times a_{h w(e i x o)}$

Como foram realizadas medições em três amostras de cada tipo de instrumento rotatório, fez-se a média quadrática dos valores do nível global de vibrações de cada instrumento rotatório, resultando em um valor médio de nível global de vibrações para cada um deles $\left(a_{h v m}\right)$.

0 cálculo da exposição diária às vibrações é dado pela Equação 3:

$A_{8}=a_{h v m} \sqrt{\frac{T}{T_{0}}}$

sendo:

- $a_{h v m}$ o nível global de vibrações médio de cada tipo de caneta;

- $T_{0}$ a duração de referência (8 horas);

- $T$ o tempo de exposição diária.

\section{Resultados e discussão}

Nos gráficos a seguir foram reportados os resultados do processamento do sinal de aceleração. Nas Figuras 5 a 8 a representação da aceleração no domínio da frequência é dada na forma de bandas de 1/3 de oitava, já incluída a função de ponderação. 0 valor do nível global de vibrações ( $\left.a_{h v}\right)$ é resumido na Figura 9.

Além da $a_{h v m}$ deve ser conhecido o tempo diário de exposição às vibrações $(\mathrm{T})$. Dessa forma, calcula-se a exposição diária $A_{8}$, conforme a Equação 3. Adotou-se um tempo diário de 6 horas, representando uma rotina intensa de trabalho. Os valores resultantes da exposição estão mostrados na Tabela 1. A caneta de alta rotação foi a que apresentou o menor nível de vibrações transmitidas ao operador.

A forma de avaliação sugerida pela ISO 5349 acerca do valor de $A_{8}$ é reproduzida na Equação 4 e na Figura 10.

$D_{y}=31,8 A_{8}^{-1,06}$

$D_{y}$ é o período de exposição total ao longo da vida do operador, em anos.

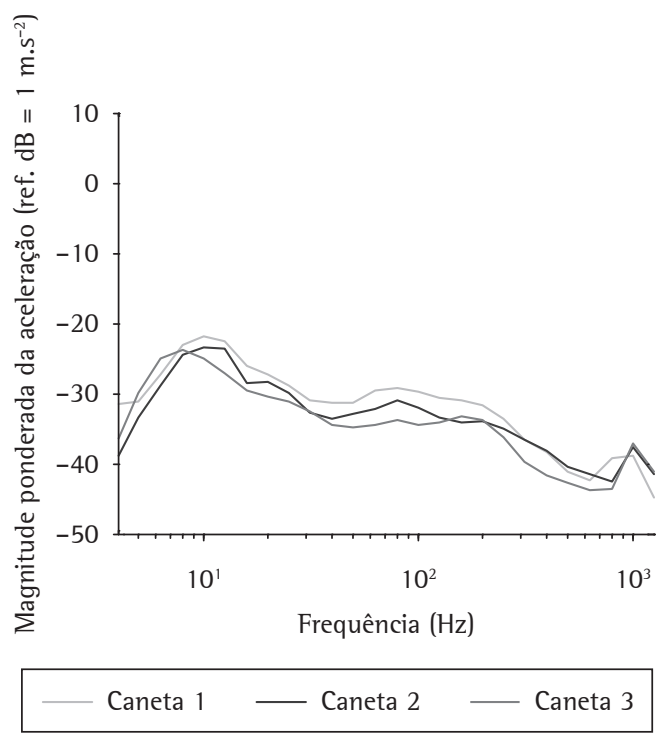

Figura 5. Canetas de alta rotação.

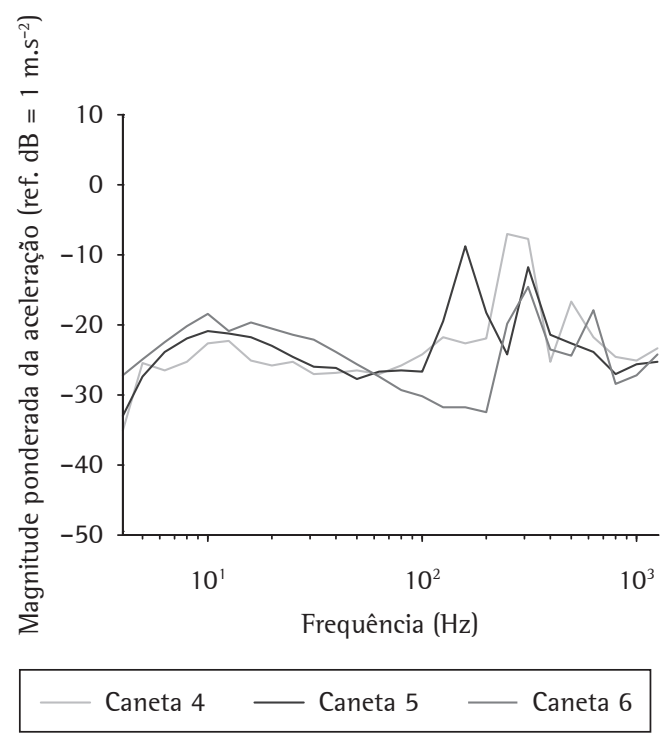

Figura 6. Canetas de baixa rotação.

Percebe-se que valores de exposição menores do que $2 \mathrm{~m} . \mathrm{s}^{-2}$ sequer foram levados em consideração nos estudos reportados pela 1SO. Aplicando-se a exposição da caneta para peça de mão na Equação 4, tem-se como resultado um período de 17 anos para ocorrer danos em 10\% das pessoas. Entretanto, deve-se ressaltar, de acordo com Szymmanska (2001) e Balbinot e Tamagna (2001 apud SEBASTIÃO; MARZIALE; ROBAZZI, 2007), que equipamentos de baixa frequência, entre 5 e $20 \mathrm{~Hz}$, são potencialmente mais perigosos que os que emitem alta frequência. 


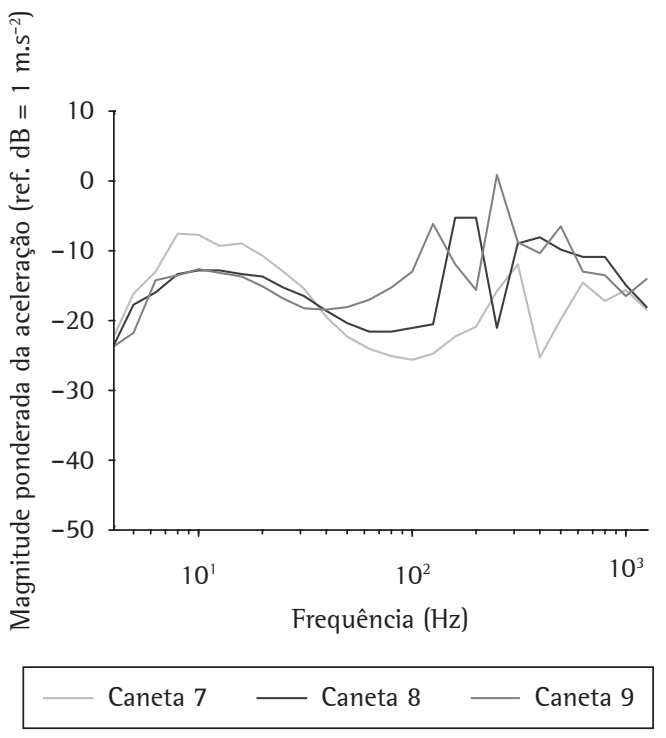

Figura 7. Contra-ângulo de baixa rotação.

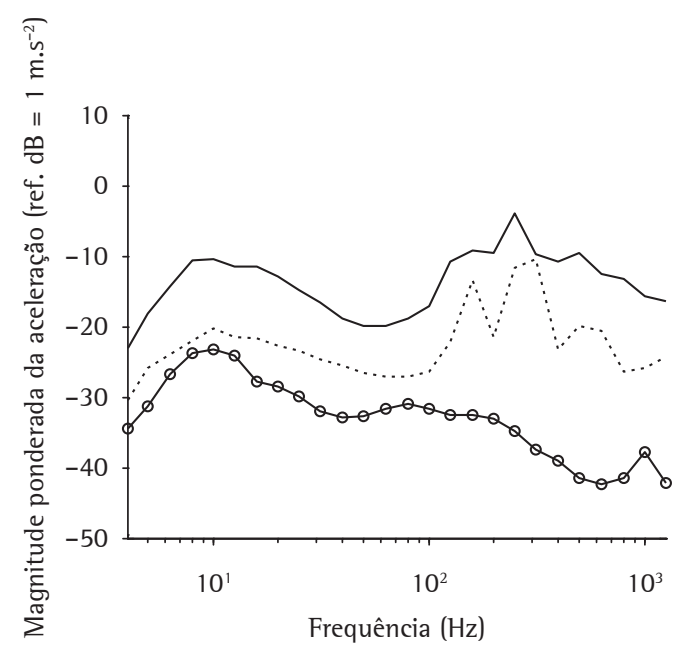

$$
\begin{aligned}
& \text { - C Caneta de alta-rotação } \\
& \text { … Caneta de baixa-rotação } \\
& \text { — Contra-ângulo de baixa-rotação }
\end{aligned}
$$

Figura 8. Comparação entre os diversos tipos de canetas (valores médios).

Para Bernard (1997), na maioria dos estudos encontra-se taxa de prevalência maior do que $20 \%$ entre trabalhadores expostos à síndrome da vibração mão/braço, mas isso não quer dizer que os valores encontrados no presente estudo não sejam importantes, tendo em vista que para Regis Filho, Michels e Sell (2006) há associação estatisticamente significante entre os dois sexos para presença de LERs/DORTs, sendo que para Sebastião, Marziale e Robazzi (2007) a vibração é um agente de risco que atua em concordância com outros.

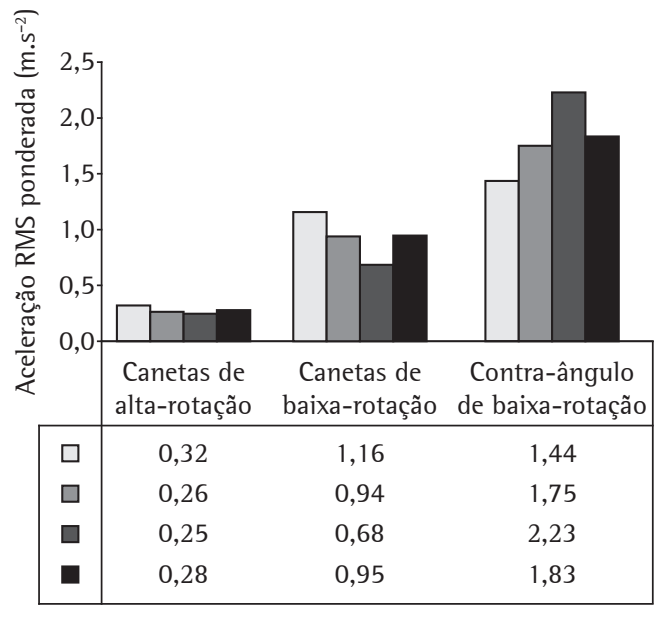

$\square$ Amostra $1 \square$ Amostra $2 \square$ Amostra 3

Média

Figura 9. Aceleração RMS ponderada.

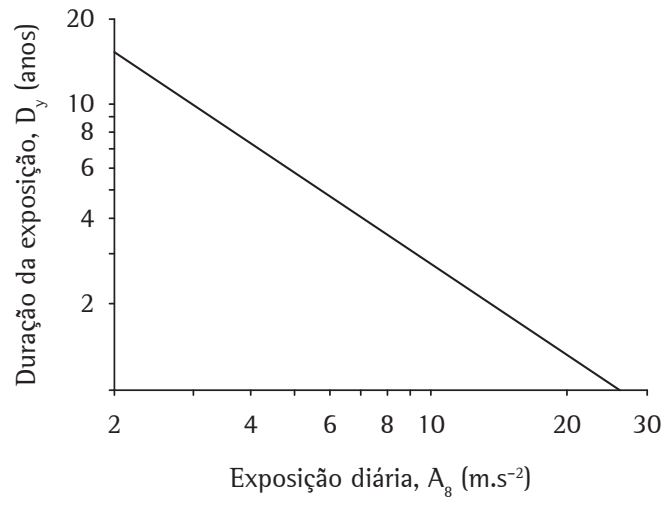

Figura 10. Exposição limite a vibrações para ocorrência do fenômeno de dedos brancos em 10\% dos trabalhadores.

Tabela 1. Valor da exposição diária a vibrações localizadas $\left(A_{8}\right)$.

\begin{tabular}{ccc}
\hline Instrumento & $\mathrm{a}_{\mathrm{hvm}}\left(\mathrm{m} \cdot \mathrm{s}^{-2}\right)$ & $\mathrm{A}(8)\left(\mathrm{m} \cdot \mathrm{s}^{-2}\right)$ \\
\hline Caneta de alta rotação & 0,28 & 0,24 \\
Caneta de baixa rotação & 0,95 & 0,82 \\
Caneta para peça de mão & 1,83 & 1,59 \\
\hline
\end{tabular}

Por outro lado, os resultados também estão abaixo do estipulado pela Diretiva Europeia 2002/44, que fixa um teto de $5 \mathrm{~m} \cdot \mathrm{s}^{-2}$ como limite de exposição diário $\left(A_{8}\right)$ e $2,5 \mathrm{~m} \cdot \mathrm{s}^{-2}$ como nível de ação. Entretanto, é necessário ressaltar que segundo Regis Filho, Michels e Sell (2006), a região de punho/mão, com 18,30\%, é a segunda mais afetada pelas LERs/ DORTs, sendo ombro/braço com 39,40\% a primeira, e que está de acordo com Lundborg et al. (1987), que afirmam haver evidência razoável suportando uma associação entre exposição à vibração e síndrome do túnel carpal. 
Dados cinemáticos, dos estudos de Regis Filho, Michels e Sell (2005), revelaram que as principais atividades realizadas pelos cirurgiões-dentistas submetem duas ou mais regiões do corpo a posturas consideradas de risco médio e alto de ocorrência de LERs/DORTs, bem como a análise dos sinais eletromiográficosindica um grandecomprometimento dos grupamentos musculares flexores e extensores do carpo e do trapézio - isso explica a maior incidência das LERs/DORTs nas regiões de ombro/braço e punho/mão, atuando a vibração nessa última região como fator coadjuvante deletério ao da má postura com desvio ulnar do punho.

\section{Conclusão}

0 presente estudo indica que somente a utilização dos instrumentos rotatórios avaliados, usados pelos cirurgiões-dentistas, não representaria risco ocupacional em termos das vibrações transmitidas às mãos, pois seria necessário que fossem operados por seis horas contínuas de trabalho ao longo de 17 anos para ocorrer danos em 10\% das pessoas. Entretanto, deve-se considerar que cirurgiões-dentistas trabalham mais de seis horas diárias, embora com interrupções, têm uma vida profissional produtiva de mais de 35 anos e que $10 \%$ dos integrantes de uma categoria profissional é um percentual bastante significativo.

Por outro lado, diversos estudos indicam que a maioria dos cirurgiões-dentistas, em virtude da utilização de instrumentos que não obedecem a critérios ergonômicos e da realização de tarefas inadequadamente prescritas, entre outros fatores, enfrenta condições adversas de trabalho, onde dor e desconforto e o desenvolvimento de LERs/DORTs estão presentes, e vibrações transmitidas às mãos, através dos instrumentos rotatórios avaliados, podem ser fator coadjuvante significativo para o aparecimento das LERs/DORTs, mais precisamente da síndrome do túnel carpal, o que demonstra a importância e significância dos resultados encontrados.

0 conjunto de efeitos fisiológicos das vibrações leva a crer que fortes oscilações mecânicas diminuem a capacidade de desempenho e concentração e em muitas situações aumentam o risco de falhas e acidentes.

\section{Referências}

ARMSTRONG, T. J. et al. Ergonomics and the effects of vibration in hand-intensive work. Scandinavian Journal of Work Environment \& Health, v. 13, p. 286-289, 1987.
BURDORF, A.; MONSTER, A. Exposure to vibration and selfreported health complaints of riveters in the aircraft industry. The Annals Occupational Hygiene, v. 35, p. 287-298, 1991.

BERNARD, B. P. Musculoskeletal disorders (MSDs) and workplace factors. Atlanta: CDC, 1997. Disponível em: $<$ http://www.cdc.gov/niosh>.

CÂNDIDO, L.; BITTENCOURT, M. Z.; REGIS FILHO, G. I. Lesões por esforço repetitivo em cirurgiões-dentistas: um estudo de caso. Cisto sinovial de punho. Jornal Brasileiro de Clínica Odontológica Integrada, v. 7, n. 42, p. 463-466, 2003.

CARNICELLl, M. V. F. Exposição ocupacional à vibração transmitida através das mãos: uma revisão sobre 0 distúrbio vascular periférico. Revista Brasileira de Saúde Ocupacional, v. 22, n. 82, p. 35-44, 1994.

FADEL, M. A. V. et al. Lesões por esforço repetitivo em cirurgiões-dentistas: um estudo de caso: dedo em gatilho. Revista da Associação Paulista de Cirurgióes Dentistas, v. 60, supl., p. 189, 2006.

GEMNE, G. Pathophysiology and multifactorial etiology of acquired vasospastic disease (Raynaud Syndrome) in vibration exposed workers. Scandinavian Journal of Work Environment \&t Health, v. 8, p. 243-249, 1982.

GEMNE, G.; LUNDSTROM, R.; HANSSON, J. Disorders induced by work with hand-held vibrating tools: a review of current knowledge for documentation. Arbete Och Hãlsa, v. 6, p. 1-83, 1993.

GRANDJEAN, E. Manual de ergonomia: adaptando o trabalho ao homem. Porto Alegre: Bookman, 1998. 338 p.

GUO, H. R. et al. Back pain among workers in the United States: national estimates and workers at high risk. American Journal of Industrial Medicine, v. 28, n. 5, p. 591-602, 1995.

INTERNATIONAL ORGANIZATION FOR STANDARDIZATION - 1S0. 1SO5349-1. Mechanical vibration: measurement and evaluation of human exposure to hand-transmitted vibration. Switzerland, 2001.

IWATA, H.; MAKIMO, S. Prevalence of Raynaud's phenomenon in individuals not using vibrating tools. Sangro lgaku, v. 29, p. 500-503, 1987.

LIIRA, J. P. et al. Long-term back problems and physical work exposures in the 1990 Ontario Health Survey. American Journal Public Health, v. 86, n. 3, p. 382-387, 1996.

LUNDBORG, G. et al. Intraneural edema following exposure to vibration. Scandinavian Journal of Work Environment \& Health, v. 13, n. 4, p. 326-329, 1987.

LUNDGORG, G. et al. Vibration exposure and peripheral nerve fiber damage. Journal Hand Surgery, v. 15A, n. 2, p. 346-351, 1990.

LUOPAJÄRVl, T. et al. Prevalence of tenosynovits and other injuries of the upper extremities in repetitive work. Scandinavian Journal of Work Environment \& Health, v. 5, supl. 3, p. 48-55, 1979.

MARTINS, C. A.; FADEL, M. A. V.; REGIS FILHO, G. 1. Lesões por esforço repetitivo em cirurgiões-dentistas: um estudo de caso - síndrome do túnel carpal. Jornal Brasileiro de Clínica Odontológica Integrada, v. 7, n. 41, p. 363-367, 2003.

NECKING, L. E. et al. Vibration-induced muscle injury: an experimental model and preliminary findings. Journal Hand Surgery British, v. 17B, p. 270-274, 1992. 
NEPOMUCENO, L. X. As vibrações mecânicas como agentes de insalubridade. Revista Brasileira de Saúde Ocupacional, 1984.

REGIS FILHO, G. 1.; MICHELS, G.; SELL, 1. Lesões por esforços repetitivos em cirurgiões-dentistas: aspectos epidemiológicos, biomecânicos e clínicos. Itajaí: Editora da Universidade do Vale do Itajaí, 2005. p. 280. (v. 1)

REGIS FILHO, G. 1.; MICHELS, G.; SELL, I. Lesões por esforços repetitivos/distúrbios osteomusculares relacionados ao trabalho em cirurgiões-dentistas. Revista Brasileira de Epidemiologia, v. 9, n. 3, p. 346-359, 2006.

SEBASTIÃO, B. A.; MARZIALE, M. H. P.; ROBAZZI, M. L. C. Uma revisão sobre os efeitos adversos ocasionados na saúde de trabalhadores expostos à vibração. Revista Baiana de Saúde Pública, v. 31, n. 1, p. 178-186, 2007.
STENLUND, B. et al. Shoulder tendinitis and its relation to heavy manual work and exposure to vibration. Scandinavian Journal of Work Environment \& Health, v. 19, p. 43-49, 1993.

STENLUND, B. et al. Radiographic osteoarthrosis in the acromioclavicular joint resulting from manual work or exposure to vibration. British Journal of Industrial Mededicine, v. 49, p. 588-593, 1992.

TAKEUCHI, T. et al. Pathological changes observed in the finger biopsy of patients with vibration-induced white finger. Scandinavian Journal of Work Environment \& Health, v. 12, p. 280-283, 1986.

VIIKARI-JUNTURA, E. et al. Prevalence of epicondylitis and elbow pain in the meat-processing industry. Scandinavian Journal of Work Environment \&t Health, v. 17, n. 1, p. 38-45, 1991.

\title{
Occupational exposure of dental surgeons to mechanical vibration: a case report
}

\begin{abstract}
The objective of this study was to estimate exposure to vibration caused by the use of rotary instruments by dentists. The rotary instruments were analyzed at high- and low-speed rotation. The deleterious effects of vibration on the hand-arm assembly are a function of frequency and amplitude of the signal. 1t was observed that the effects depend on whether the excitement consists of a single frequency or presents a complex spectrum. We tested three samples of each rotating device (pen), namely: high-speed pen, counter-angle and low-speed hand piece, also low-spin. The use of rotary instruments by dentists was evaluated; there is an some occupational risk in terms of vibration to the hands, especially when operated continuously for six hours, which may be an important contributing factor in the development of Cumulative Trauma Disorders (CTD)/Work Related Musculoskeletal Disorders (WRMD). Moreover, the range of physiological effects of vibration suggests that fluctuations impair the mechanical performance and in many cases increase the risk of error and accidents.
\end{abstract}

\section{Keywords}

Vibrations. Occupational exposure. Surgeon-dentists. 